\title{
A CONSISTENT NONRELATIVISTIC QUARK MODEL WITH DYNAMICAL PION EXCHANGE
}

\author{
S. AICHER and M. BRACK \\ Niels Bohr Institute, Blegdamsvej 17, DK-2100 Copenhagen, Denmark \\ and Institut für Theoretische Physik, Universität Regensburg, D-8400 Regensburg, Fed. Rep. Germany '
}

Received 12 February 1988

\begin{abstract}
We investigate the nonrelativistic quark model with a residual pion-quark interaction of the Weinberg-Schwinger type including p-wave and s-wave coupling. The pion-nucleon vertex is renormalised to third order in the pion-quark coupling constant $f_{\pi}$ and adjusted to reproduce the empirical pion-nucleon coupling strength, leading to an axial charge $g_{\mathrm{A}}=1.42$ and a value of $f_{\pi}=98.8 \mathrm{MeV}$. Including the pion exchange currents dynamically through one-pion loops, we reproduce the nucleon magnetic moments within less than $10 \%$ for any quark core radius $0.3 \mathrm{fm} \leqslant r_{\mathrm{q}} \leqslant 0.7 \mathrm{fm}$. All these results change by less than $5 \%$ if the pion mass is varied from zero to its physical value.
\end{abstract}

Several attempts have recently been made [1-7] to add pion exchange effects to the nonrelativistic quark model (NRQM). The formal motivation for this is to approximately incorporate chiral symmetry, which in principle can be done also for consitituent quarks [8]. A practical motivation comes from the application of the NRQM to baryon spectroscopy. Indeed, the inclusion of pion exchange as an additional residual interaction between the quarks - beside the one-gluon exchange (OGE) - helps to explain [5,7] the experimentally known smallness of the spin-orbit splittings in the baryon spectrum (especially in the non-strange sector). Since the pion exchange also contributes to the nucleon (N)-delta $(\Delta)$ splittings, the quark-gluon coupling constant $\alpha_{\mathrm{s}}$ can hereby be considerably reduced [4,5] (to $\alpha_{\mathrm{s}} \simeq 0.35$ ) compared to the value $(\simeq 1)$ necessary for fitting the ground state $\mathrm{N}-\Delta$ splitting by the OGE alone [9] which leads to far too strong spin-orbit splittings.

The aim of the present paper is to apply the NRQM with dynamical pion exchange to electromagnetic properties of the nucleons. At the same time we shall bring the model closer to chiral symmetry by including an swave type second-order pion-quark coupling. The value of the pion-quark coupling constant (which was an adjustable parameter in refs. $[4,5])$ is fixed to reproduce the empirical pion-nucleon coupling after a renormalization of the pion-nucleon vertex by pion loop corrections. We shall show that this model is practically equivalent to the cloudy bag model (CBM) [10] and leads to reasonable values of the nucleon magnetic moments and the axial charge $g_{\mathrm{A}}$ rather independently of the quark core radius.

We use the following pion-quark interaction:

$\mathscr{H}_{\pi \mathrm{q}}=\psi^{+}(\boldsymbol{r})\left[\left(1 / 2 f_{\pi}\right) \boldsymbol{\sigma} \cdot \mathrm{i} \nabla \boldsymbol{\tau} \cdot \boldsymbol{\phi}(\boldsymbol{r})-\left(1 / 2 f_{\pi}\right)^{2} \boldsymbol{\tau} \cdot \boldsymbol{\phi}(\boldsymbol{r}) \times \mathrm{i} \partial_{0} \boldsymbol{\phi}(\boldsymbol{r})\right] \psi(\boldsymbol{r})$,

where $\psi(\boldsymbol{r})$ is the quark wave function of the NRQM and $\phi(\boldsymbol{r})$ the quantized pion field; $f_{\pi}$ is the coupling constant which will be determined below. The interaction (1) has the usual p-wave part and an s-wave part which is nonlinear in the pion field. Its relativistic equivalent (the "Weinberg lagrangian" [11]) can be derived from a chirally invariant nonlinear coupling of pions to massless quarks by a local chiral rotation of the quark spinors and expansion up to second order in the pion field. (See ref. [12] where this procedure was used to

\footnotetext{
1 Permanent address.
} 
derive the "volume coupled" version of the CBM.) In order to determine now the value of $f_{\pi}$, we shall relate it to the pion-nucleon vertex. The general $\pi \mathrm{B}_{1} \mathrm{~B}_{2}$ vertex is $\left\langle\mathrm{B}_{2}\left|\sum_{i=1}^{3} \mathscr{H}_{\pi \mathrm{q}}(i)\right| \pi \mathrm{B}_{1}\right\rangle$, where $\left|\mathrm{B}_{1}\right\rangle,\left|\mathrm{B}_{2}\right\rangle$ are NRQM three-quark baryon states. This vertex contains a structure form factor $\exp \left(-q^{2} / 6 \alpha_{0}^{2}\right)$ which is just the Fourier transform of the gaussian factor in the quark wave functions. Hereby $q$ is the momentum of the incoming pion and $\alpha_{0}$ is the inverse rms radius of the nucleon in the ground state (the "quark core radius" $r_{\mathrm{q}}$ ). In the diagrams below, this vertex shall be denoted by an empty blob. Since we are going to include pionic loop corrections to the electromagnetic nucleon vertex below, it is consistent to add such corrections also to the $\pi \mathrm{B}_{1} \mathrm{~B}_{2}$ vertices. This leads, besides renormalizing the coupling strength, to a form factor $F_{\mathrm{nB}_{1} \mathrm{~B}_{2}}\left(q^{2}\right)$ shown in the diagrams by a dashed blob. To determine $f_{\pi}$ and $F_{\pi \mathrm{B}_{1} \mathrm{~B}_{2}}\left(q^{2}\right)$ consistently, we solve iteratively the following coupled integral equations:

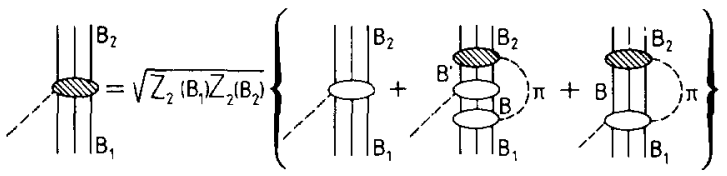

in which $F_{\pi \mathrm{BB}}\left(k^{2}\right)$ appears on the RHS at the dashed vertices under the momentum $(\boldsymbol{k})$ integrals coming from the pion loops. (See ref. [13] for the explicit form of the equations and for calculational details.) In eq. (2), $Z_{2}^{\pi}(B)$ is the wave function renormalization factor (see, e.g., ref. [10]) of the baryon B defined by $Z_{2}^{\pi}(\mathrm{B})=1 /\left[1-\left.(\partial / \partial E) \sum_{\mathrm{B}}^{\pi}(E)\right|_{E=M_{\mathrm{B}}}\right]$ in terms of the pionic self energy correction $\sum_{\mathrm{B}}^{\pi}$ (see, e.g., refs. $[6,10]$ ). We use only one form factor in eq. (2), as well as in $\Sigma_{\mathrm{B}}^{\pi}$, to avoid double counting of the leading terms of order $\left(f_{\pi}\right)^{4}$ implicitly included.

In the iterative solution of eq. (2), we restrict the summation over the intermediate states $B, B^{\prime}$ to the ground state $\mathrm{N}$ and $\Delta$ only, although reasonable convergence can be obtained [6,13] including higher resonances with the form factors we obtain in this way. The loops in eq. (2) and $\Sigma_{\mathrm{B}}^{\pi}$ are calculated in usual nonrelativistic perturbation theory (using the physical masses of $\mathrm{N}$ and $\Delta$ and including their recoil in the determinators); summation over all possible time orderings is understood in all diagrams. In each iteration, the form factors are fitted for $q \leqslant q_{\max }=0.8 \mathrm{GeV}^{-1}$ to a monopole from

$F_{\pi \mathrm{B}_{1} \mathrm{~B}_{2}}\left(q^{2}\right)=1 /\left[1+\left(q / A_{\pi \mathrm{B}_{1} \mathrm{~B}_{2}}\right)^{2}\right]$.

(The results depend to less than $10 \%$ on the precise value of $q_{\max }$ up to which the monopole fit is done.) The value of $f_{\pi}$ entering at each vertex on the RHS of eq. (2) is determined by equating the LHS of eq. (2) at $q=0$ with the renormalized value $f_{\pi}^{*}$ which is chosen to reproduce the experimentally known $\pi N N$ coupling constant

$g_{\pi \mathrm{NN}}=13.45, \quad f_{\pi}^{*}=5 M_{\mathrm{N}} / 3 g_{\pi \mathrm{NN}}=116.2 \mathrm{MeV}$.

This leads to a cubic equation for the unrenormalized $f_{\pi}$ to be solved at each iteration. After convergence we find $f_{\pi}=98.8 \mathrm{MeV}$ when using the standard value $\alpha_{0}=426 \mathrm{MeV}$ (corresponding to a quark core radius $\left\langle r^{2}\right\rangle^{1 / 2}=r_{\mathrm{q}}=1 / \alpha_{0}=0.46 \mathrm{fm}$ ) which ensures a correct spectroscopy [4,5]. Note that this value of $f_{\pi}$ differs only by $6 \%$ from the empirical value of the pion decay constant $f_{\pi}=93.3 \mathrm{MeV}$. The corresponding values of $A_{\pi \mathrm{B}_{1} \mathrm{~B}_{2}}$ in the monopole form factors (3) are

$A_{\pi \mathrm{NN}}=1.77 \mathrm{GeV}, \quad A_{\pi \mathrm{N} \Delta}=1.54 \mathrm{GeV}, \quad A_{\pi \Delta \Delta}=1.22 \mathrm{GeV}$.

(Note that if the structure form factor $\exp \left(-q^{2} / 6 \alpha_{0}^{2}\right)$ were included in the monopole fit, one would approximately find $0.90,0.86$ and $0.79 \mathrm{GeV}$, respectively, for the three cutoffs in eq. (5).) With the above values for $f_{\pi}$ and the form factors, we checked the convergence of eq. (2) with respect to inclusion of higher resonances in the intermediate states. Even though these were shown to affect strongly the self energies $\Sigma_{B}^{\pi}$ in ref. [6], we found here that the $Z_{2}^{\pi}$ (B) change much less. The resulting value of $f_{\pi}$ varies, in fact, less than $5 \%$ and the $Z_{2}^{\pi}$ (B) less than $10 \%$ upon including intermediate states up to the $N=2$ harmonic oscillator shell, thus justifying our truncation of the model space to $\mathrm{N}$ and $\Delta$ ground states. 


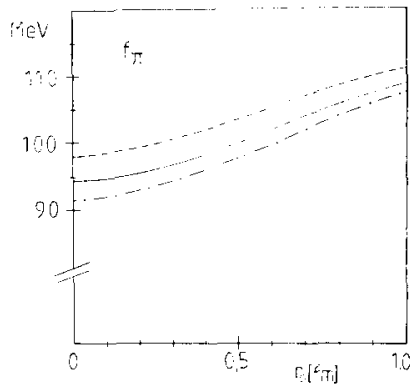

Fig. 1. Coupling constant $f_{\pi}$ versus quark core radius $r_{\mathrm{q}}$. Solid line: including s-wave interaction, $m_{\pi}=138 \mathrm{MeV}$; dashed line: without s-wave interaction, $m_{\pi}=138 \mathrm{MeV}$; dash-dotted line: including s-wave interaction, $m_{\pi}=0$.

The result for $f_{\pi}$ depends also very little on the choice of the quark core radius, i.e. the constant $\alpha_{0}$ in the wave functions. In fig. 1 we show the results for $f_{\pi}$ versus $r_{\mathrm{q}}=1 / \alpha_{0}$. Also shown there is the result obtained when the s-wave part of the interaction (1) is omitted. We see thus that its inclusion brings us indeed much closer to the experimental value of $f_{\pi}$ which would be that of a chirally symmetric model. Another sign of the closeness of chiral symmetry is the result (shown by a dash-dotted line) obtained in the soft-pion limit, i.e. taking the pion mass $m_{\pi}$ to be zero. The behaviour of the curves in fig. 1 can be easily analyzed in a simplified "toy model" in which the pionic form factors (3) are put to unity, recoil and $N-\Delta$ mass difference in the intermediate states are ignored and the pion mass is zero. All the loop integrals can then be done analytically and the cubic equation for $f_{\pi}$ following from eq. (2) becomes

$\frac{1}{f_{\pi}^{*}}=\frac{1}{f_{\pi}}\left\{\frac{4 \pi^{2}+\frac{45}{8}\left(\alpha_{0} / f_{\pi}\right)^{2}}{4 \pi^{2}+\frac{57}{8}\left(\alpha_{0} / f_{\pi}\right)^{2}}\right\}=\frac{1}{f_{\pi}} Z_{1}^{\pi}(\mathrm{N}) Z_{2}^{\pi}(\mathrm{N})$,

where $Z_{1}^{\pi}(\mathrm{N})$ is the vertex renormalization factor accounting for the diagrams on the RHS of eq. (2) for the $\pi \mathrm{NN}$ vertex at $q=0$. Eq. (6) becomes linear in the two limits $\alpha_{0}=0$ (infinite radius) and $\alpha_{0}=\infty$ (zero radius); the solution for $f_{\pi}$ thus has to move between the limits $f_{\pi}^{*}$ and $\frac{45}{57} f_{\pi}^{*}$, respectively. The same factors as in eq. (6) also renormalize the axial charge: $g_{\mathrm{A}}=\frac{5}{3} Z_{1}^{\pi}(\mathrm{N}) Z_{2}^{\pi}(\mathrm{N})$, leading to $g_{\mathrm{A}}=\frac{5}{3} \cdot \frac{45}{57}=\frac{25}{19}=1.31$ for zero radius and to the usual (unrenormalized) nonrelativistic value of $\frac{5}{3}$ for an infinite radius. (Without the s-wave pion-quark interaction, one would obtain $\frac{5}{3} \cdot \frac{49}{57}=1.43$ for zero radius.) In our full calculation corresponding to the solid line in fig. 1 , we get $Z_{1}^{\pi}(\mathrm{N})=1.52, Z_{2}^{\pi}(\mathrm{N})=0.561$ and thus $g_{\mathrm{A}}=1.42$ for the standard radius of 0.46 fm. Our results can easily be seen to fulfill the Goldberger-Treiman relation to within less than $10 \%$ for quark core radii $r_{\mathrm{q}} \leqslant 0.7$ fm.

It is very interesting that in the CBM [10] with linearized $\gamma_{5}$ coupling, the same toy model calculation ("soft$\pi$ " limit, no form factors and degenerate $\mathrm{N}, \Delta$ without recoil) leads to exactly the same equation (6), replacing 45 by 49 in the numerator, since there is no s-wave quark coupling in this version of the CBM, and replacing $\alpha_{0}^{-2}$ by $\frac{2}{3} R^{2}$, where $R$ is the bag radius. This shows that our results are very little dependent on the fact that we use massive quarks and nonrelativistic wavefunctions, once the quark core radius is suitably chosen. Indeed, the quark mass has not entered our model so far; the only parameter besides $f_{\pi}$ is the constant $\alpha_{0}$. The relativistic quark spinors in the CBM only give rise to a different structure form factor (a Bessel function instead of a gaussian; in fact, in practical calculations in the CBM [10] a gaussian was used, too, for numerical convenience). After integration in the toy model limit, the same algebraical coefficients appear in eq. (6) besides a rescaling of the quark core radius. (A similar analysis can also be made for the nucleon magnetic moments [13].)

We now turn to the discussion of the electromagnetic form factors of the nucleon. To second order in the pion-quark coupling, they are given by the following set of diagrams: 


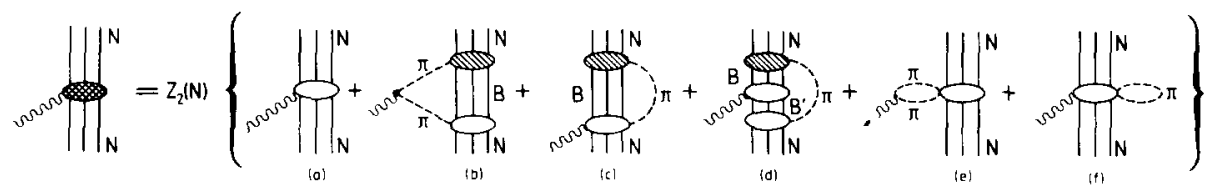

which are obtained from the interaction (1) by minimal coupling to the electromagnetic field (see ref. [13] for calculational details). The cross-hatched blob at the vertex on the LHS of eq. (7) signifies the (nonrelativistic) electromagnetic form factors $G_{\mathrm{E}, \mathrm{M}}^{T}\left(q^{2}\right)$ ( $T=0$ and 1 for isoscalar and isovector case, respectively). On the RHS the renormalized coupling constant $f_{\pi}^{*}$ is used everywhere; the dashed blobs include the selfconsistently obtained form factors (3), (5). The $G_{\mathrm{E}}^{T}\left(q^{2}\right)$ should be normalized to $G_{\mathrm{E}}^{0}(0)=G_{\mathrm{E}}^{1}(0)=e / 2$, where $e$ is the unit charge. It is, indeed, straightforward to see that the isoscalar charge, to which only diagrams (a) and (d) in eq. (7) contribute, is exactly $e / 2$, i.e. the isoscalar electric current is exactly conserved. (Hereby it is important to include the same number of pion-quark form factors in the corresponding loops of eqs. (2), (7) and in $\Sigma_{\mathrm{B}}^{\pi}$.) The isovector charge, however, to which also diagrams (b) and (c) contribute (the contributions of (e) and (f) cancel exactly), turns out to deviate by about $3 \%$ from the value $e / 2$. We see thus that our interaction hamiltonian does not conserve exactly the isovector electric current. The violation is, however, small and we correct it by renormalizing ad hoc the isovector charge to $e / 2$. (We should like to emphasize at this point that violation of the isovector charge conservation also occurs in the CBM as used in ref. [10].)

After this renormalization, we obtain the magnetic moments of the nucleons from $G_{M}^{0}(0)=\left(\mu_{\mathrm{p}}+\mu_{\mathrm{n}}\right) / 2$ $G_{\mathrm{M}}^{1}(0)=\left(\mu_{\mathrm{p}}-\mu_{\mathrm{n}}\right) / 2$. Since these moments are expressed in units of $e \hbar / 2 m_{\mathrm{q}} c$, they depend explicitly on the constituents quark mass $m_{\mathrm{q}}$. In the simple NRQM without poins, one has $\mu_{\mathrm{p}}=e \hbar / 2 m_{\mathrm{q}} c$ (and $\mu_{\mathrm{n}}=-\frac{2}{3} \mu_{\mathrm{p}}$ ); the quark mass is therefore chosen to be $m_{\mathrm{q}}=330 \mathrm{MeV}$ approximately. Here, however, the "quark core term" (diagram (a) in eq. (7)) is reduced by the factor $Z_{2}^{\pi}(N)<1$. On the other hand, the diagrams (b), (c) and (d) also contribute positively to $\mu_{\mathrm{p}}$, bringing the total magnetic moment back to approximately the experimental one, similary as in the CBM case [10]. It might be interesting to note that in the static one-pion-exchange approximation, we find that the pion exchange currents (sum of Seagull and pole terms) contribute much less to the magnetic moment and depend rather sensitively on the value of the pion mass. For $m_{\pi}=0$ they give about $13 \%$ of the isovector magnetic moment (see also ref. [1]); with $m_{\pi}=138 \mathrm{MeV}$ they cancel to about $3 \%$. In contrast to this, the dynamical pion exchange through diagrams (b), (c), (d) in eq. (7) gives about 35-45\% of the total $\mu_{\mathrm{N}}$ (for $r_{\mathrm{q}} \simeq 0.5 \mathrm{fm}$ ), rather independently of the pion mass.

Our resulting magnetic moments (in units of the experimental $\mu_{\mathrm{p}}$ ) are shown in fig. 2 as functions of the quark core radius $r_{\mathrm{q}}=1 / \alpha_{0}$. The solid lines give the results including only ground state $\mathrm{N}$ and $\Delta$ as intermediate states in the loops in eq. (7); in the dashed lines the contributions of all intermediate states up to the $N=2$

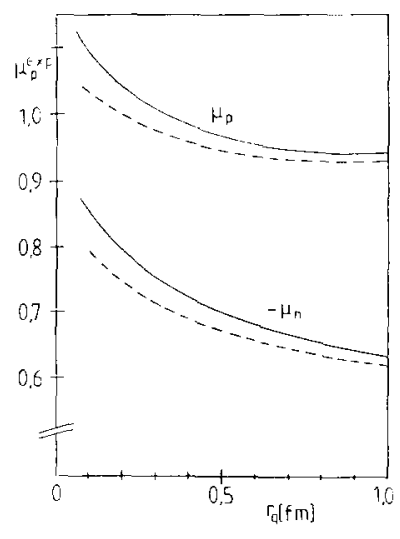

Fig. 2. Proton and neutron magnetic moments (in units of experimental proton moment ) versus quark core radius $r_{\mathbf{q}}$. solid lines: including only the $N=0$ oscillator shell; dashed lines: including $N=0,1,2$ oscillator shells for the intermediate states. 
harmonic oscillator shell are included. Again a good convergence is found for the summation over intermediate states. The constituents quark mass has been chosen to be the standard value $m_{\mathrm{q}}=330 \mathrm{MeV}$. The results do not depend strongly on the quark core radius; for $r_{\mathrm{q}} \geqslant 0.25 \mathrm{fm}$ we reproduce the experimental magnetic moments within about $10 \%$. We thus come to the interesting result that the sum of quark core and pionic contributions to the magnetic moments has the right magnitude and depends little on the core radius within reasonable limits, a result which is also found in relativistic chiral quark models [10,14]. Compared to the latter, our model has the advantage that the CM motion is treated correctly and that the convergence of the summation over intermediate states has been ensured without any ad hoc cutoff. (In the CBM calculatations [10] only ground state $\mathrm{N}$ and $\Delta$ were included; in ref. [14], where pion loops were calculated on the quark level, only intermediate $1 \mathrm{~s}$ and $1 \mathrm{p}$ states were included.)

For the mean squared charge radii we find with $m_{\mathrm{q}}=330 \mathrm{MeV}$ and $\alpha_{0}=426 \mathrm{MeV}$ the following results:

$\left\langle r^{2}\right\rangle_{\mathrm{E}}^{\mathrm{p}}=(0.67 \mathrm{fm})^{2},\left\langle r^{2}\right\rangle_{\mathrm{E}}^{\mathrm{n}}=-(0.30 \mathrm{fm})^{2}$.

(It should be mentioned that the loops in diagrams (e) and (f) of eq. (7), which contribute to the isovector charge radii, diverge. We have regularized them with a monopole form factor with cutoff $\lambda \simeq 4 \pi f_{\pi} \simeq 1 \mathrm{GeV}$ (see ref. [8]); their contribution to the radii in eq. (8) then is less than $3 \%$.) If we include the empirical charge form factor of the pion at the $\gamma \pi \pi$-vertices in eq. (7), we obtain the following results:

$\left\langle r^{2}\right\rangle_{\mathrm{E}}^{\mathrm{p}}=(0.71 \mathrm{fm})^{2}, \quad\left\langle r^{2}\right\rangle_{\mathrm{E}}^{\mathrm{n}}=-(0.34 \mathrm{fm})^{2}$,

which are in fair agreement with experiment. (The pionic charge radius can, in priniciple, be obtained from the assumption of vector meson dominance; we feel, however, that we would overstress our model by also including $\rho$-mesons.)

In summary, we can say that we obtain very reasonable electromagnetic properties of the nucleons in our NRQM using the pion-quark interaction (1) and including pion loops. Note that we could fine-tune our results by changing the quark mass $m_{\mathrm{q}}$ and/or choosing an appropriate oscillator parameter $\alpha_{0}$ (i.e. quark core radius). We want, however, to keep the spectroscopy of the baryon resonances in mind, which is the particular strength of the NRQM. We therefore have demonstrated that we can obtain reasonable results with values of $m_{\mathrm{q}}$ and $\alpha_{0}$ that are compatible with baryon spectroscopy $[4,5]$.

As already noted in ref. [6], the dynamical pion exchange alone does not yield the full experimental $N-\Delta$ splitting of $293 \mathrm{MeV}$, if the pion-quark coupling constant is adjusted to the empirical pion-nucleon coupling constant as we have done it here. In fact, with the present results of eqs. (2)-(5) we find a pionic contribution to this splitting (including only $\mathrm{N}$ and $\Delta$ intermediate states and the standard parameters $m_{\mathrm{q}}=330 \mathrm{MeV}, \alpha_{0}=426$ $\mathrm{MeV})$ of $\operatorname{Re}\left(\Sigma_{\Delta}^{\pi}\right)-\Sigma_{\mathrm{N}}^{\pi}=220 \mathrm{MeV}$. This splitting is smaller than the empirical one; it would be further reduced by higher intermediate states [6]. In order to obtain the correct $N-\Delta$ splitting, one still has to include a weak residual OGE interaction; in first-order perturbation we would need a quark-gluon coupling constant $\alpha_{\mathrm{s}} \simeq 0.25$ 0.35 to account for the full splitting. Note that this is in line with the value $\alpha_{s} \simeq 0.28$ obtained in ref. [8]. This residual OGE interaction will, of course, also affect the electromagnetic form factors of the nucleon (see, e.g., ref. [15]) and thus modify our above results. A new calculation of the baryon spectrum, taking pion and gluon exchange into account and aiming at a simultaneous fit of electromagnetic properties of the nucleons and the baryonic excitation spectrum, is in progress [16].

In conclusion, it appears possible to formulate an approximately chiral NRQM with a residual interaction dominated by dynamical pion exchange, yielding satisfactory electromagnetic properties of the nucleons and applicable to baryon spectroscopy due to a correct treatment of the centre of mass. The parameters of the model are: (1) the pion-quark coupling constant $f_{\pi}$ which can be taken to be the empirical pion decay constant; (2) the constituent quark mass $m_{\mathrm{q}}$ of the order of (but not necessarily equal to!) $330 \mathrm{MeV}$ to give the correct nucleon magnetic moments; (3) the harmonic oscillator constant $\alpha_{0}=\sqrt{\hbar \omega m_{\mathrm{q}}}$ of the order $400-450 \mathrm{MeV}$, corresponding to an oscillator frequency $\hbar \omega=500-550 \mathrm{MeV}$, to give the correct gross structure of the baryon spectrum; and (4) an OGE coupling constant $\alpha_{\mathrm{s}}$ of the order of $0.25-0.35$ to obtain a reasonable fine structure of the spectrum. 
We have greatly benefitted from stimulating discussions with P. Guichon, G. Ripka and M. Schaden. We acknowledge several enlightening discussions with R.K. Bhaduri and M.V.N. Murthy during stays at McMaster University which were made possible by NATO Grant No. RG86/0074 for international collaboration in basic science research. We also thank N. Kaiser, M. Rho, W. Weise and A. Wirzba for valuable comments. The hospitality at the Niels Bohr Institute, where this work was completed, is highly appreciated.

\section{References}

[1] G.E. Brown, M. Rho and V. Vento, Phys. Lett. B 97 (1980) 423.

[2] Y. Nogami and N. Ohtsuka, Phys. Rev. D 26 (1982) 261;

K.G. Horacsek, Y. Iwamura and Y. Nogami, Phys. Rev. D 32 (1985) 3001.

[3] J. Navarro and V. Vento, Nucl. Phys. A 440 (1985) 617, and earlier references quoted therein.

[4] M.V.N. Murthy and R.K. Bhaduri, Phys. Rev. Lett. 54 (1985) 745.

[5] M.V.N. Murthy, M. Brack, R.K. Bhaduri and B.K. Jennings, Phys. C 29 (1985) 385; M.V.N. Murthy, R.K. Bhaduri and E. Tabarah, Z. Phys. C 31 (1986) 81.

[6] M. Brack and R.K. Bhaduri, Phys. Rev. D 35 (1987) 3451.

[7] W. Blask, M.G. Huber and B. Metsch, Z. Phys. A 326 (1987) 413.

[8] A. Manohar and H. Georgi, Nucl. Phys. B 234 (1984) 189.

[9] N. Isgur and G. Karl, Phys. Rev. D 18 (1978) 4187; D 19 (1979) 2653; see also: N. Isgur, in: XVI School of Subnuclear physics (Erice, 1978), ed. A. Zichichi (Plenum, New York, 1980 ) p. 107.

[10] S. Théberge and A.W. Thomas, Nucl. Phys. A 393 (1983) 252; see also A.W. Thomas, Advances in nuclear physics, eds. J.W. Negele and E. Vogt, Vol. 13, (Plenum, New York, 1983 ) p. 1.

[11] S. Weinberg, Phys. Rev. Lett. 18 (1967) 188; see also J. Schwinger: Particles and sources (Gordon and Breach, New York, 1969).

[12] A.W. Thomas, J. Phys. G 7 (1981) L283.

[13] S. Aicher and M. Brack, to be published.

[14] M. Oleszczuk, M. Schaden and E. Werner, Nucl. Phys. A 445 (1985) 639.

[15] N. Isgur, G. Karl and D.W.L. Sprung, Phys. Rev. D 23 (1981) 163, see also earlier references quoted therein.

[16] S. Aicher, M. Brack, H. Genzken and M.V.N. Murthy, to be published. 